\title{
Estructuras II (Grado en Edificación): aprender de la práctica
}

\section{Structures II (Degree on Building): learning from practice}

ESPERANZA RODRÍGUEZ MAYORGA

ORCID: https://orcid.org/0000-0002-2721-5154

Universidad de Sevilla.

Dpto. Estructuras de Edificación

e Ingeniería del Terreno.

espe@us.es

Fecha de recepción: 23/07/19

Fecha de aceptación: 19/11/19

DOI: http://dx.doi.org/10.12795/9788447221912.066 Pp.: 1519-1545 
La asignatura Estructuras II se imparte en el tercer curso del Grado en Edificación. Su programa es amplio, abarcando el dimensionado de estructuras de hormigón armado y de acero laminado. La asignatura consiste en el primer contacto de los alumnos con las estructuras de hormigón armado pero el único con las estructuras de acero laminado. Las principales dificultades que los alumnos presentan son: (i) el manejo de la normativa de obligado cumplimiento; y (ii) el paso de la teoría a la práctica. La experiencia que aquí se describe se diseñó en un intento por minimizar ambos problemas. Como parte de este ciclo de mejora de asignatura completa dentro de la fase de permanencia del Programa de Formación en Innovación Docente del Profesorado (FIDOP), se reorganizó el temario de la asignatura completa en torno a dos proyectos de arquitectura reales. De esta forma, el objetivo del curso se centró en el dimensionado y cálculo de sendas estructuras para estos proyectos facilitando, de este modo, el aprendizaje a partir de una práctica similar a la práctica profesional.

Palabras Clave: Estructuras II, grado en Edificación, docencia universitaria, experimentación docente universitaria, talleres.

\section{Abstract}

The subject Structures II is a taught in the third year of the degree on Building. Its wide syllabus encompass the design and analysis of reinforced concrete structures and Steel structures. Mainly, the difficulties that students usually find are: (i) dealing with the codes; and (ii) passing from theory to practice. The experience described in this paper addresses both problems. This experience consisted of the organisation of the whole subject around two real architecture projects. Finally, the subject was targeted to design and analyse two structures for these buildings. This way, learning emerges from the practice.

Keywords: Structures II, degree on Building, university teaching, experimentation in university teaching, workshops. 


\section{Introducción}

El ciclo de mejora que se describe en esta experiencia se enmarca dentro del programa de Formación e Innovación Docente del Profesorado (FIDOP) que desarrolla en Secretariado de Formación y Evaluación de la Universidad de Sevilla junto con el Instituto de Ciencias de la Educación (ICE). Concretamente, dentro de la fase de permanencia (REFID) de dicho programa. El Ciclo de Mejora que aquí se describe abarca una asignatura completa, Estructuras II, que se imparte en el primer cuatrimestre del tercer curso del grado en Ingeniería de la Edificación. Ninguna experiencia similar se había desarrollado con anterioridad en referencia a esta asignatura.

Estructuras II es una asignatura obligatoria del Grado en Edificación (Plan de Estudios 2017). Su carga horaria semanal es de cuatro horas, distribuida en dos bloques horarios de dos horas cada uno, impartidos en dos días diferentes durante las quince semanas de las que consta el curso. Le corresponden, por tanto 6 créditos ECTS. Estructuras II se imparte seguidamente a la asignatura Estructuras I (segundo cuatrimestre del segundo curso) y seguida por la asignatura Estructuras III (segundo cuatrimestre del tercer curso). La formación en las disciplinas relativas a las Estructuras se completa con tres asignaturas optativas situadas en el segundo cuatrimestre del cuarto curso. Un breve recorrido por la trayectoria de un alumno supone que se introduce en las Estructuras sentando las bases del análisis estructural (esfuerzos, tensiones y deformaciones), continúa después analizando y dimensionando estructuras de acero y hormigón en Estructuras II para terminar profundizando, en la tercera de las asignaturas obligatorias, en el diseño, análisis y dimensionado de una estructura real de hormigón armado. En parte de esta última asignatura, se utilizan programas comerciales de cálculo de estructuras. Un alumno interesado en temas relativos a las estructuras que escogiese las asignaturas 
optativas además profundizaría en cálculo sísmico, rehabilitación de estructuras y análisis y dimensionado de estructuras y cimentaciones usando programas informáticos.

Así pues, la asignatura que ocupa este ciclo de mejora docente consta de la aplicación de los principios teóricos adquiridos en el primero de los cursos a los dos materiales más utilizados en estructuras: acero laminado y hormigón armado. Esta segunda asignatura obligatoria es de especial importancia debido principalmente a un aspecto: constituye prácticamente el único contacto que los alumnos tienen con el dimensionado y análisis de las estructuras de acero laminado, incluyendo aquellos que escojan las tres asignaturas optativas ofertadas en el departamento. Éste es uno de los factores que hacen especialmente importante maximizar el aprendizaje del alumnado.

La asignatura Estructuras II es la asignatura de aquellas impartidas por el departamento en la Escuela Técnica Superior de Ingeniería de la Edificación con peores resultados en cuanto a número de aprobados, tasa de abandono, etc. En general, la asignatura resulta extensa y abstracta, y presenta dificultades para su comprensión principalmente debido a la complejidad que introducen el manejo de la normativa vigente (España. Ministerio de la Vivienda, 2011; Fomento, 2008) y la necesaria aplicación de los conocimientos adquiridos en la asignatura inmediatamente anterior (Estructuras I), y que, muchos estudiantes, no han logrado aprobar. El objetivo de este ciclo de mejora docente, es hacer una primera mejora que abarque toda la asignatura, ordenando los contenidos de acuerdo a un esquema lógico en referencia al mapa conceptual de la asignatura, asi como replantear la metodología docente empleada. De este modo, se considera que se facilitará la comprensión de la asignatura por parte del estudiante.

Jornadas de Formación e Innovación Docente del Profesorado | № 2 (2019) Esta obra se distribuye con la licencia Creative Commons Reconocimiento-NoComercial-SinObraDerivada Internacional (CC BY-NC-ND 4.0.) 


\section{Contexto de la intervención}

La asignatura Estructuras II se imparte en grupos reducidos. Concretamente, este ciclo de mejora se ha aplicado en el grupo 3, con docencia en martes y jueves, de 17.00 a 19.00. El número de alumnos matriculados es de 35 , de los cuales han asistido regularmente en torno a 20. Al ser un grupo de tarde, el perfil de los alumnos, en su mayoría, es de personas que compaginan la actividad laboral con el estudio del Grado. Una importante ventaja que presenta este grupo en concreto es que la totalidad de ellos está ocupada en trabajos relacionados con el sector de la construcción. Por el contrario, prácticamente todos presentan en común el no haber superado recientemente la asignatura de Estructuras I, bien por no tenerla aprobada aun, bien por tenerla convalidada o aprobada antes de su incorporación al mundo laboral. El perfil de los alumnos encuestados (aproximadamente los que asistieron regularmente a clase) queda resumido en la Figura 1.

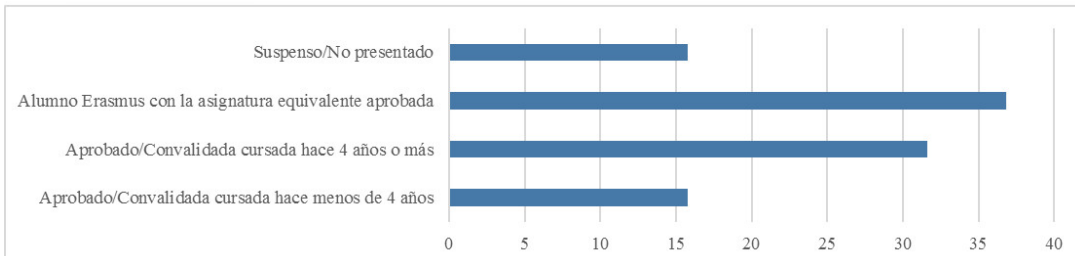

Figura 1. Características del alumnado en porcentaje en referencia a la asignatura Estructuras I (inmediatamente anterior a Estructuras II) durante el curso 2019-20

Estructuras II es una asignatura particular en cuanto a su programación. Los contenidos se dividen en dos grandes bloques, denominados "Análisis de estructuras de hormigón" y "Análisis de piezas metálicas". Ambos bloques se imparten simultáneamente, asignándole a cada uno de ellos una sesión semanal de dos horas. Los dos bloques son impartidos, evaluados y calificados independientemente, en la mayoría de las veces incluso por diferentes profesores. En esta experiencia, la misma profesora se ha hecho cargo de un grupo completo, es decir, de los 
dos bloques temáticos del mismo grupo. El programa de la asignatura contempla la existencia de 52 horas presenciales teórico prácticas y 90 horas no presenciales de trabajo autónomo del estudiante. 8 horas presenciales del alumno serán destinadas a la realización de exámenes teórico-prácticos. El programa establece como metodología docente las clases expositivas, el desarrollo de trabajos, proyectos, problemas y ejercicios, la exposición de trabajos y debates, y los seminarios y las conferencias para las horas presenciales. En las horas no presenciales, la metodología a seguir por los estudiantes será el desarrollo de trabajos, proyectos, problemas y ejercicios.

El desarrollo habitual del curso consiste en el desarrollo de 26 sesiones de dos horas cada una de actividades teórico-prácticas donde prima como metodología la clase expositiva y la resolución de problemas y otras dos sesiones en las que se realizan los dos exámenes parciales de evaluación. Esta programación se realiza para cada uno de los dos bloques temáticos de la asignatura, resultando así el cómputo final de horas que se ha indicado anteriormente.

\section{Diseño previo del Ciclo de Mejora}

\section{Modelo Metodológico}

De acuerdo a lo expuesto en el apartado anterior, el modelo metodológico más cercano a la práctica habitual sería (Porlan, 2017):

\section{TEORÍA $\rightarrow$ PROBLEMA $\rightarrow$ CONCLUSIONES}

Donde hay una primera exposición teórica que se aplica a un problema, en el que reside la mayor responsabilidad del aprendizaje del alumno, y sobre el que se obtienen unas conclusiones. 
El diseño de este ciclo de mejora busca alcanzar el modelo:

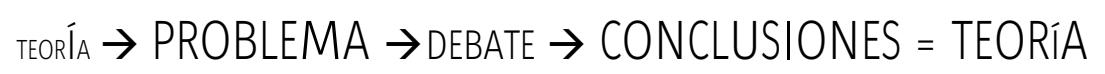

Es decir, partir de la teoría, para pasar a la aplicación de la misma en la resolución de un problema. Tras el debate y la puesta en común de los resultados, volver sobre la teoría inicial como parte de las conclusiones de los problemas resueltos. Es de recalcar que, durante el diseño del Ciclo de Mejora, se pretendía que en el debate se involucrara el aprendizaje autónomo de los alumnos durante su horario no presencial. Esto no ha sido factible, debido principalmente al hecho de que los alumnos no han llevado el trabajo no presencial al día, es decir, han llevado varias semanas de retraso en su desarrollo. Igualmente, el debate ha sido dificultoso de conseguir en la mayoría de los casos. Finalmente, el modelo llevado a cabo ha sido:

\section{PROBLEMA $\rightarrow$ TEORÍA $\rightarrow$ SOLUCIÓN DEL PRO- BLEMA $\rightarrow$ CONCLUSIONES = TEORÍA}

Modelo que ha resultado bastante positivo, principalmente debido al planteamiento del problema a resolver con antelación al estudio de la teoría necesaria para resolverlo. Esto se ha hecho a dos niveles: en primer lugar, se ha planteado un problema real y global de curso que el diseño de una estructura real; en segundo lugar, este problema global se ha descompuesto en otros subproblemas de menor entidad (dimensionado de vigas, pilares, forjados, etc.) abordables en un periodo de tiempo menor, y a resolver como unidades más o menos independientes durante una o dos clases presenciales. 


\section{Principios didácticos}

Los principios didácticos con los que se aborda este ciclo de mejora parten de facilitar el paso de la teoría a la práctica y de estimular el trabajo no presencial del alumno. Así pues: la reorganización de la asignatura se ha llevado a cabo en base a los principios didácticos:

- Fomentar la creatividad de los estudiantes, aumentando de este modo el interés de los mismos en la asignatura. Se ha huido de los problemas establecidos por el profesor, han sido los alumnos los que han propuesto problemas para posteriormente resolverlos. Asimismo, se ha puesto en crisis la creatividad del alumno, encorsetándola dentro de la realidad fisica de las estructuras, es decir, han debido crear algo construible. En caso de no hacerlo, los alumnos lo han identificado por sus propios medios al tratar de resolver un problema sin solución física posible.

- Favorecer el aprendizaje autónomo del estudiante en la medida de lo posible. Para ello, se han establecido unas pautas temporales y de contenido al trabajo no presencial. Se ha pretendido que el estudiante asimilase el contenido de las clases teóricas mediante su aplicación a la práctica autónoma. Esto no ha sido totalmente posible.

- Saber trabajar en grupo, lo que difiere considerablemente de trabajar en grupo. Ello supone saber integrarse en un equipo de trabajo cualquiera, en ocasiones con desconocidos, resultar productivo para el equipo y saber resolver sus diferencias enfocando su relación a la búsqueda del bien colectivo.

- Establecer una vía de retroalimentación del profesor a través de cuestionarios o de la observación directa en clase. De esta forma, el profesor ha conocido de primera mano lo que el alumno ha entendido de lo que el profesor ha expuesto. Esto ha permitido al profesor comprender los mapas mentales de los alumnos 
y pudiendo individualizar de este modo el proceso de enseñanza-aprendizaje, reorientando y completando las clases cuando ha sido necesario.

- Trabajar en clase, no ser un mero espectador. Así los alumnos han aprendido haciendo, no viendo lo que el profesor hace. Se ha desarrollado un esquema temporal que ha permitido que primero el alumno observe y luego repita de forma consciente.

\section{Descripción del diseño}

El diseño inicial del ciclo de mejora que ocupa este documento partió de la reorganización completa de los contenidos recogidos en el programa de la asignatura, y su articulación en base a un proyecto real de un edificio. En paralelo, la parte no presencial de la asignatura se organizó siguiendo el mismo esquema. Para esta reorganización de la asignatura se ha tomado como patrón el mapa conceptual del proceso de cálculo de una estructura, mapa conceptual que es propuesto ya en la asignatura de Estructuras 1 (Rodriguez Mayorga, 2018) y que exclusivamente incluye contenidos de carácter básico (Figura 2).

Jornadas de Formación e Innovación Docente del Profesorado | № 2 (2019) Esta obra se distribuye con la licencia Creative Commons Reconocimiento-NoComercial-SinObraDerivada Internacional (CC BY-NC-ND 4.0.) 


\section{ESPERANZA RODRÍGUEZ MAYORGA}
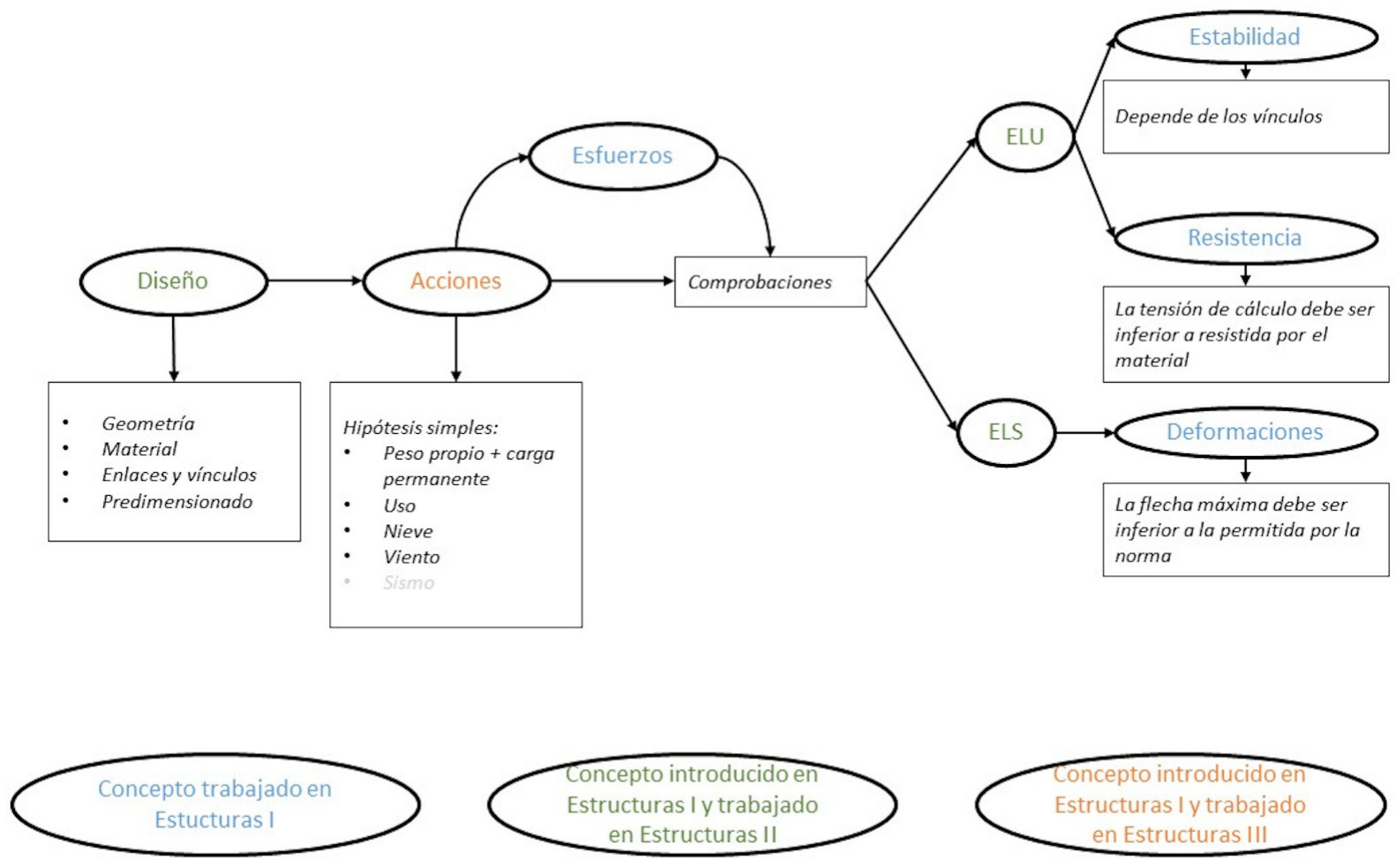

Aclaraciones y contenido procedimental

Figura 2. Mapa conceptual propuesto para la asignatura Estructuras I y tipos de contenido incluidos en el mismo.

Jornadas de Formación e Innovación Docente del Profesorado | № 2 (2019)

(ㅇ) $\mathbb{Q} \otimes$ Esta obra se distribuye con la licencia Creative Commons

Reconocimiento-NoComercial-SinObraDerivada

Internacional (CC BY-NC-ND 4.0.) 


\section{ESPERANZA RODRÍGUEZ MAYORGA}

Una vez particularizado para la asignatura de Estructuras II, y concretamente para los dos bloques temáticos de "Estructuras de hormigón armado" y "Estructuras de acero laminado", resulta en los mapas de contenidos de las figuras 3 y 4.

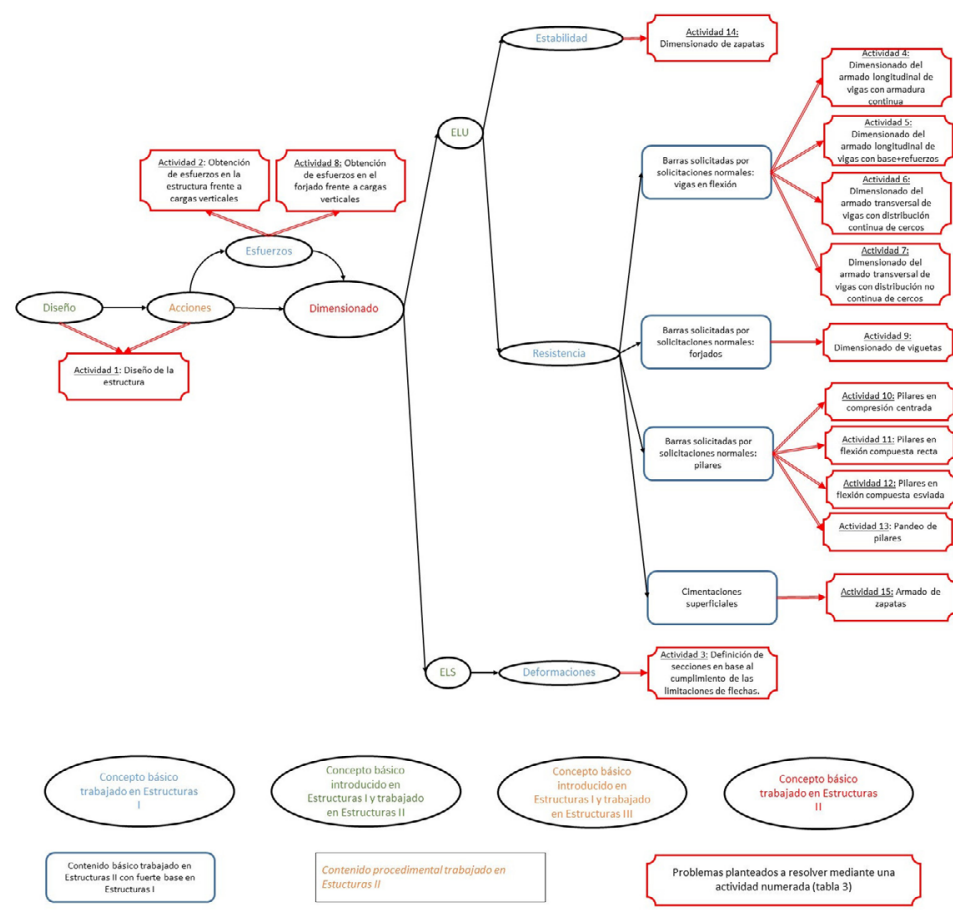

Figura 3. Mapa de contenidos del bloque temático I: Estructuras de hormigón armado y tipos de contenido incluidos en el mismo.

Jornadas de Formación e Innovación Docente del Profesorado | № 2 (2019)

(c) $($ ) $\Theta$ Esta obra se distribuye con la licencia Creative Commons 


\section{ESPERANZA RODRÍGUEZ MAYORGA}

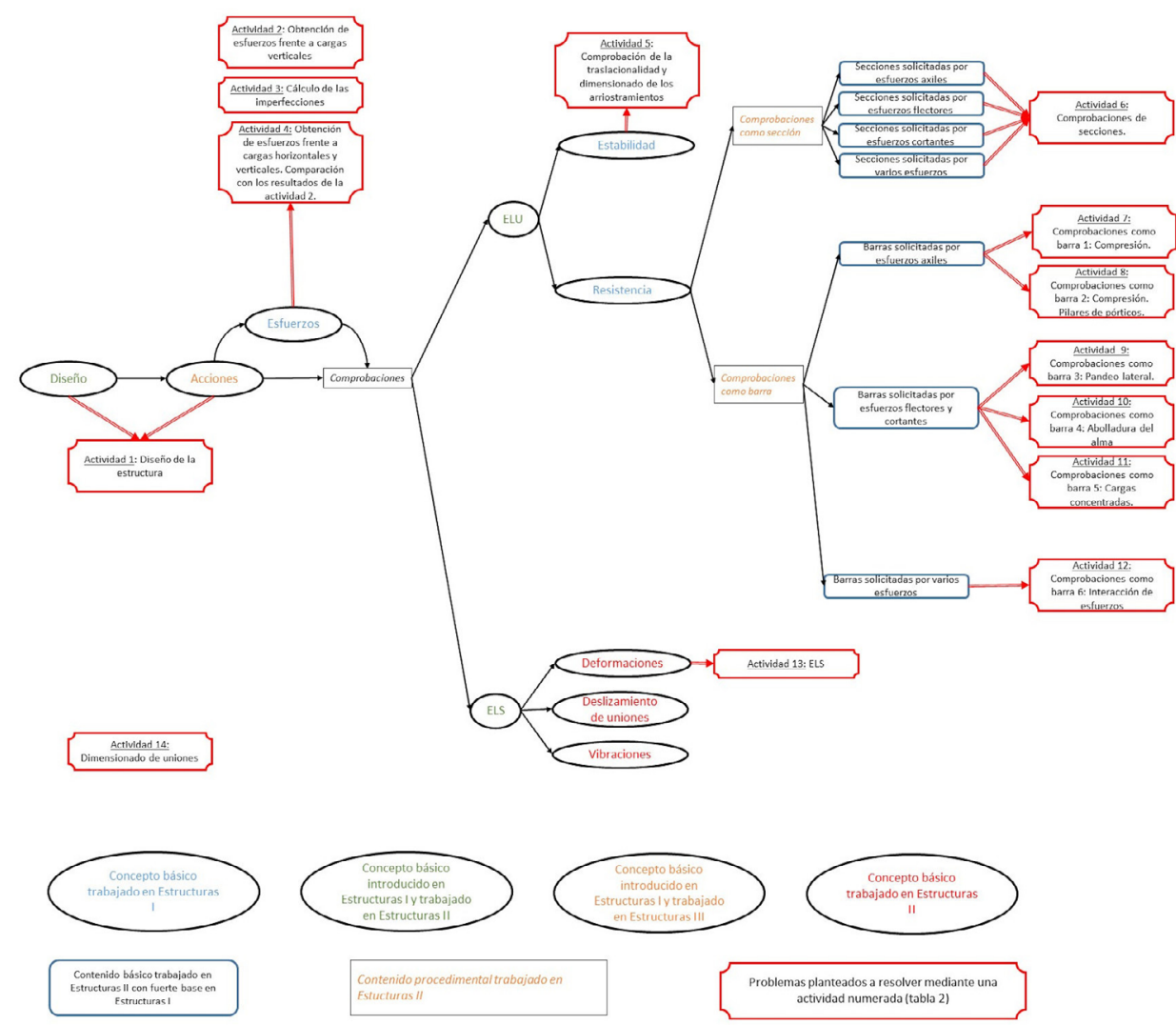

Figura 4. Mapa de contenidos del bloque temático II: Estructuras de acero laminado y tipos de contenido incluidos en el mismo.

Jornadas de Formación e Innovación Docente del Profesorado | № 2 (2019)

(C) Esta obra se distribuye con la licencia Creative Commons

cc) (i) $\Theta$ Reconocimiento-NoComercial-SinObraDerivada

Internacional (CC BY-NC-ND 4.0.) 
Con la articulación del curso en torno a la resolución de dos proyectos reales de arquitectura, los alumnos funcionarán como "pequeños estudios de arquitectura" que deberán resolver un problema que se le plantea: el diseño y dimensionado de la estructura de un proyecto de edificación. El primer proyecto, el proyecto de curso (Proyecto Glass Cabin del estudio de arquitectura atelierRISTING IIc (AtelierRISTING, 2018) servirá como ejemplo durante las clases presenciales, y todas las actividades del curso (figuras 3 y 4) se realizarán sobre sus elementos estructurales. En paralelo, los alumnos desarrollarán un proyecto en horario no presencial(Alison Burke, 2011; Kozlowski \& Ilgen, 2006), que se ha denominado proyecto de grupo (Proyecto Casa Caja del estudio de arquitectura S-AR ((S-AR, 2015))), sobre el que deberán resolver los mismos problemas que se han planteado en clase y desarrollar las actividades del curso (figuras 3 y 4 ) de manera autónoma. Para este fin, los alumnos han trabajado en equipos de 3 a 5 alumnos y han desarrollado, principalmente en horario no presencial, el proyecto de la estructura de un edificio sencillo propuesto por el profesor, utilizando como materiales el hormigón armado y el acero laminado. Han diseñado, por tanto, cuatro estructuras correspondientes a los dos proyectos y a los dos materiales mencionados anteriormente. Esos dos grandes problemas se han dividido en otros problemas de menor entidad que se corresponden con las actividades numeradas en las figuras 3 y 4 . Así pues, estas actividades están diseñadas para, en su conjunto, resolver el problema general del curso, que es el diseño y dimensionado de cada uno de los proyectos que se plantean.

\section{Aplicación del Ciclo de Mejora}

Tal y como se ha planteado, en el diseño de la docencia se ha recurrido a un modelo que ha permitido una concepción de clases más dinámicas. Aunque la intención inicial fuera la de desarrollar la práctica totalidad del curso 
mediante clases participativas por parte del alumnado, las limitaciones temporales existentes obligaron a desarrollar de esta forma solo una parte del curso. Finalmente, las clases expositivas ocuparon gran parte del curso. Independientemente de este hecho las clases no consistieron en meras exposiciones del docente, sino que se promovió la participación del alumno. Asignatura pendiente ha resultado la realización de debates en clase, pues, aunque sean una herramienta potente para el aprendizaje de los alumnos (Bain \& Barberá, 2007), en varias ocasiones se han intentado iniciar de manera poco exitosa. Por el contrario, los alumnos se han mostrado receptivos en todo momento, interviniendo cuando se les ha solicitado y aportando cuanto estaba en su mano.

La organización del curso en torno a proyectos de arquitectura se acerca al estudiante a la realidad de la práctica profesional. Las actividades que se han ido realizando han ido surgiendo de la necesidad de avanzar en el diseño de un Proyecto de Ejecución completo. Debido a las limitaciones temporales, solo la primera parte del curso ha sido incluida en este documento. A lo largo de la experiencia se desarrollaron actividades de varios tipos:

- Planteamiento de problemas: el curso se ha articulado en torno a los subproblemas con los que se encuentra un profesional que tiene que calcular una estructura (dimensionado de vigas, definición de modelos de cálculo, etc.)

- Clases expositivas: orientadas a la resolución del problema planteado.

- Aplicación práctica: principalmente interpretación y aplicación de la normativa.

- Talleres: en total, se realizaron cuatro experiencias basadas en los talleres conceptuales (Finkel, 2000), aunque su desarrollo final no coincida exactamente con la definición de esta actividad (Rodríguez-Mayorga, Jiménez-Alonso, Pachón-García, Cámara, \& Compán, 2018). 
Todas las actividades desarrolladas en este marco se encuentran recogidas en las tablas 1 y 2 , así como al problema que dan respuesta. Posteriormente se describe detalladamente el desarrollo de los cuatro talleres que se han desarrollado hasta el momento.

Tabla 1. Resumen de problemas planteados durante las clases, actividades desarrolladas para dar solución a los mismos y conocimientos teóricos aportados en el Bloque I (hormigón armado).

\begin{tabular}{|c|c|c|c|}
\hline Semana & $\begin{array}{l}\text { Problemas } \\
\text { planteados }\end{array}$ & Actividades asociadas & Teoría asociada \\
\hline 1 & $\begin{array}{l}\text { Obtener esfuerzos } \\
\text { en 2D. } \\
\text { Diseñar una } \\
\text { estructura } \\
\text { unidireccional de } \\
\text { malla de barras con } \\
\text { pilares y vigas de } \\
\text { hormigón armado }\end{array}$ & $\begin{array}{l}\text { Calculo manual de } \\
\text { esfuerzos en vigas } \\
\text { isostáticas; Taller 1: } \\
\text { Diseño de estructuras }\end{array}$ & $\begin{array}{c}\text { Equilibrio, esfuerzos, } \\
\text { leyes y diagramas } \\
\text { de esfuerzos, } \\
\text { redistribución de } \\
\text { esfuerzos en nudos. }\end{array}$ \\
\hline 2 & $\begin{array}{c}\text { Conocer el hormigón } \\
\text { armado como } \\
\text { material y su } \\
\text { comportamiento } \\
\text { mecánico. }\end{array}$ & $\begin{array}{l}\text { Clases expositivas: } \\
\text { Características del } \\
\text { material y de las } \\
\text { secciones. Bases de } \\
\text { cálculo; Anclajes y } \\
\text { modelos de armado }\end{array}$ & $\begin{array}{c}\text { Propiedades } \\
\text { mecánicas del } \\
\text { hormigón, del acero } \\
\text { y del hormigón } \\
\text { armado. Longitudes } \\
\text { de anclaje. }\end{array}$ \\
\hline 3 & $\begin{array}{c}\text { Identificar en qué } \\
\text { situaciones son } \\
\text { necesarios los } \\
\text { armados en vigas y } \\
\text { cómo dimensionarlos }\end{array}$ & $\begin{array}{l}\text { Clase expositiva: Armado } \\
\text { longitudinal frente a } \\
\text { solicitaciones normales; } \\
\text { Aplicación práctica: } \\
\text { armado de secciones } \\
\text { en flexión; Debate y } \\
\text { conclusiones:Relación } \\
\text { armado/esfuerzo, } \\
\text { importancia de las } \\
\text { cuantías de armado, } \\
\text { importancia del anclaje, } \\
\text { relación esfuerzo/ } \\
\text { cuantía. }\end{array}$ & $\begin{array}{l}\text { Armado longitudinal } \\
\text { de vigas en flexión }\end{array}$ \\
\hline
\end{tabular}




\begin{tabular}{|c|c|c|c|}
\hline 4 & $\begin{array}{l}\text { Identificar en qué } \\
\text { situaciones son } \\
\text { necesarios los } \\
\text { refuerzos en vigas y } \\
\text { como dimensionarlos }\end{array}$ & $\begin{array}{c}\text { Breve debate: } \\
\text { como identificar } \\
\text { en qué secciones } \\
\text { son necesarios/ } \\
\text { convenientes refuerzos. } \\
\text { Como establecer un } \\
\text { esquema de armado; } \\
\text { Aplicación práctica: } \\
\text { armado de una viga } \\
\text { siguiendo el modelo de } \\
\text { base más refuerzos. }\end{array}$ & $\begin{array}{l}\text { Armado longitudinal } \\
\text { de vigas en flexión }\end{array}$ \\
\hline 5 & $\begin{array}{l}\text { Dimensionar el } \\
\text { armado transversal } \\
\text { e identificar de qué } \\
\text { factores depende }\end{array}$ & $\begin{array}{c}\text { Clase expositiva: } \\
\text { dimensionado del } \\
\text { armado transversal en } \\
\text { la EHE 08; Aplicación } \\
\text { práctica: armado } \\
\text { transversal de una viga } \\
\text { en tramos; Conclusiones: } \\
\text { esquemas de armado, } \\
\text { interrelaciones } \\
\text { diámetro/separación/ } \\
\text { ramas }\end{array}$ & $\begin{array}{l}\text { Comportamiento del } \\
\text { hormigón a cortante, } \\
\text { armado transversal } \\
\text { de vigas en flexión }\end{array}$ \\
\hline 6 & $\begin{array}{c}\text { Cumplimiento de ELS } \\
\text { según la normativa } \\
\text { vigente. }\end{array}$ & $\begin{array}{c}\text { Taller 3: interpretación } \\
\text { de la normativa para } \\
\text { comprobación de un } \\
\text { proyecto real. }\end{array}$ & $\begin{array}{c}\text { Deformación } \\
\text { real y admisible, } \\
\text { deformación a corto y } \\
\text { a largo plazo, factores } \\
\text { que influyen en la } \\
\text { deformación }\end{array}$ \\
\hline 7 & $\begin{array}{l}\text { Recapitulación de } \\
\text { diseño estructural }\end{array}$ & $\begin{array}{c}\text { Taller 4: Diseño } \\
\text { estructural, obtención } \\
\text { de esfuerzos, } \\
\text { dimensionado de } \\
\text { armado longitudinal de } \\
\text { vigas. }\end{array}$ & $\begin{array}{l}\text { Repaso y } \\
\text { recapitulación }\end{array}$ \\
\hline
\end{tabular}


Tabla 2. Resumen de problemas planteados durante las clases, actividades desarrolladas para dar solución a los mismos y conocimientos teóricos aportados en el Bloque II (acero laminado).

\begin{tabular}{|c|c|c|c|}
\hline Semana & Problemas & Actividades asociadas & Teoría asociada \\
\hline 1 & $\begin{array}{l}\text { Esfuerzos en 3D } \\
\text { en estructuras } \\
\text { isostáticas e } \\
\text { hiperestáticas frente } \\
\text { a cargas verticales. }\end{array}$ & $\begin{array}{l}\text { Taller 2: esfuerzos } \\
\text { en estructuras: } \\
\text { programas de } \\
\text { cálculo; Debate } \\
\text { y conclusiones: } \\
\text { interrelaciones entre } \\
\text { diseño, material, tipo } \\
\text { de nudo y esfuerzos. }\end{array}$ & $\begin{array}{l}\text { Equilibrio, esfuerzos, } \\
\text { leyes y diagramas } \\
\text { de esfuerzos, } \\
\text { redistribución de } \\
\text { esfuerzos en nudos. }\end{array}$ \\
\hline 2 & $\begin{array}{c}\text { Conocer el acero } \\
\text { como material y su } \\
\text { comportamiento en } \\
\text { los rangos elásticos y } \\
\text { plásticos. }\end{array}$ & $\begin{array}{l}\text { Clases expositivas: } \\
\text { Comportamiento del } \\
\text { acero en cálculos } \\
\text { elásticos / plásticos; } \\
\text { La clasificación } \\
\text { de secciones y su } \\
\text { repercusión en el } \\
\text { cálculo de esfuerzos. }\end{array}$ & $\begin{array}{l}\text { Propiedades } \\
\text { mecánicas del acero } \\
\text { laminado, cálculo } \\
\text { elástico y plástico, } \\
\text { tipos de secciones. }\end{array}$ \\
\hline 3 & $\begin{array}{l}\text { Conocer las } \\
\text { particularidades } \\
\text { de los modelos } \\
\text { de cálculo en } \\
\text { estructuras metálicas: } \\
\text { imperfecciones y } \\
\text { traslacionalidad. }\end{array}$ & $\begin{array}{l}\text { Clases expositivas: } \\
\text { Imperfecciones; } \\
\text { Aplicación práctica: } \\
\text { Definición de las } \\
\text { imperfecciones } \\
\text { de una estructura } \\
\text { real como acciones } \\
\text { equivalentes; } \\
\text { Traslacionalidad } \\
\text { en la normativa; } \\
\text { Aplicación práctica: } \\
\text { Determinación de } \\
\text { la traslacionalidad } \\
\text { de una estructura. } \\
\text { Implicaciones } \\
\text { de los tipos de } \\
\text { arriostramientos. }\end{array}$ & $\begin{array}{c}\text { Imperfecciones en la } \\
\text { normativa, concepto } \\
\text { de traslacionalidad, } \\
\text { formas de arriostrar } \\
\text { una estructura y su } \\
\text { repercusión en su } \\
\text { comportamiento } \\
\text { estructural }\end{array}$ \\
\hline
\end{tabular}

Jornadas de Formación e Innovación Docente del Profesorado | № 2 (2019) Esta obra se distribuye con la licencia Creative Commons Reconocimiento-NoComercial-SinObraDerivada Internacional (CC BY-NC-ND 4.0.) 


\begin{tabular}{|c|c|c|c|}
\hline 4 & $\begin{array}{c}\text { Dimensionar } \\
\text { secciones en } \\
\text { estructuras de acero } \\
\text { laminado }\end{array}$ & $\begin{array}{c}\text { Clase expositiva: } \\
\text { Resistencia de } \\
\text { secciones en la } \\
\text { normativa vigente; } \\
\text { Aplicación práctica: } \\
\text { Dimensionado } \\
\text { de secciones } \\
\text { solicitadas por } \\
\text { distintos esfuerzos; } \\
\text { Conclusiones: } \\
\text { posibilidad en cuanto } \\
\text { al dimensionado } \\
\text { de estructuras } \\
\text { isostáticas frente a } \\
\text { las hiperestáticas, } \\
\text { importancia de } \\
\text { la agrupación de } \\
\text { barras para su } \\
\text { dimensionado. }\end{array}$ & $\begin{array}{l}\text { Comprobaciones } \\
\text { ELU, coeficientes } \\
\text { de seguridad, } \\
\text { comprobaciones de } \\
\text { sección, índices de } \\
\text { aprovechamiento en } \\
\text { términos de sección. }\end{array}$ \\
\hline 5 & $\begin{array}{c}\text { Comprobar barras en } \\
\text { compresión }\end{array}$ & $\begin{array}{l}\text { Clase expositiva: las } \\
\text { comprobaciones } \\
\text { de pandeo en } \\
\text { la normativa; } \\
\text { Aplicación práctica: } \\
\text { comprobación de } \\
\text { pandeo de pilares } \\
\text { estructurales; } \\
\text { Conclusiones: } \\
\text { importancia del } \\
\text { pandeo como factor } \\
\text { dimensionante en } \\
\text { estructuras esbeltas. }\end{array}$ & $\begin{array}{c}\text { Limitaciones } \\
\text { normativas de las } \\
\text { barras traccionadas } \\
\text { y comprimidas, } \\
\text { Pandeo de Euler, } \\
\text { curvas de pandeo, } \\
\text { bifurcación del } \\
\text { estado de equilibrio, } \\
\text { comprobaciones de } \\
\text { barras comprimidas. }\end{array}$ \\
\hline
\end{tabular}




\begin{tabular}{|c|c|c|c|}
\hline 6 & $\begin{array}{l}\text { Comprobar pilares } \\
\text { de pórticos en } \\
\text { compresión. } \\
\text { Solucionar problemas } \\
\text { de arriostramientos } \\
\text { en estructuras de } \\
\text { pórticos planos. }\end{array}$ & $\begin{array}{l}\text { Clase expositiva: } \\
\text { pilares de pórticos } \\
\text { en la normativa; } \\
\text { Aplicación práctica: } \\
\text { pandeo en pilares } \\
\text { de pórticos; } \\
\text { Conclusiones: } \\
\text { relación entre } \\
\text { arriostramiento / } \\
\text { longitudes de pandeo } \\
\text { / traslacionalidad } \\
\text { / índice de } \\
\text { aprovechamiento de } \\
\text { perfiles. Búsqueda } \\
\text { de soluciones a un } \\
\text { problema de fallo por } \\
\text { pandeo. }\end{array}$ & $\begin{array}{l}\text { Longitudes de } \\
\text { pandeo no canónicas, } \\
\text { influencia de la } \\
\text { rigidez de las barras } \\
\text { a flexión en la } \\
\text { longitud de pandeo, } \\
\text { arriostramientos en } \\
\text { pórticos de edificios. }\end{array}$ \\
\hline 7 & Recapitulación & $\begin{array}{l}\text { Taller 4: Diseño } \\
\text { estructural, esfuerzos, } \\
\text { dimensionado de } \\
\text { secciones, pandeo. }\end{array}$ & $\begin{array}{c}\text { Repaso y } \\
\text { Recapitulación }\end{array}$ \\
\hline
\end{tabular}




\section{Taller 1: Diseño de la estructura}

Esta primera actividad tuvo lugar el primer día de clase. Tras la toma de contacto con la asignatura, los alumnos se agruparon por parejas y plantearon un posible diseño estructural. Para ello, los alumnos contaban con información genérica facilitada por la profesora sobre luces, cantos series de perfiles, necesidad de arriostramientos, etc. De este modo, cada pareja de alumnos realizó dos diseños estructurales: uno para ser construido con hormigón armado y otro con acero laminado. Tras la puesta en común de todos los diseños planteados, se corrigieron las deficiencias e incorrecciones de cada uno de ellos llegando al diseño estructural que se calculó a lo largo del curso (Figura 5).
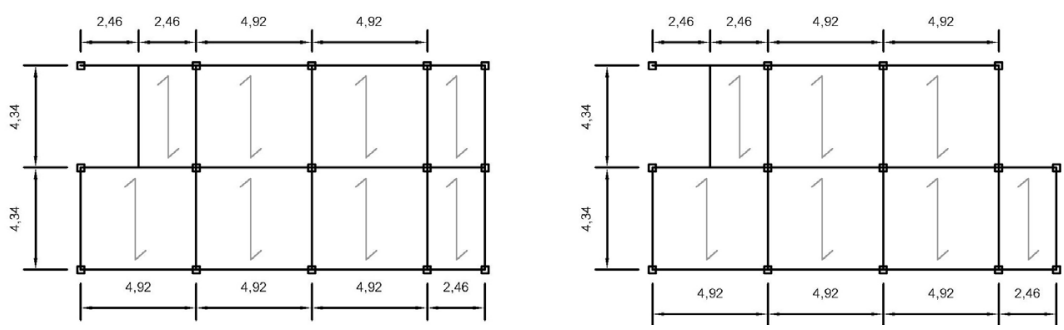

Figura 5. Planta primera (izquierda) y planta de cubiertas (derecha) diseñada como estructura para dimensionar a lo largo de curso. En azul, la localización de los arriostramientos necesarios para estabilizar la estructura en el caso de construirse con acero laminado.

\section{Taller 2: Esfuerzos}

Una vez diseñada la estructura y teniendo siempre por delante el mapa conceptual genérico de la asignatura (Figura 1), los alumnos se enfrentaron al siguiente paso, que es la obtención de los esfuerzos en la misma. Partiendo de las acciones facilitadas por la profesora, los alumnos intentaron obtener los esfuerzos por hipótesis simples en las barras de la estructura. De este modo:

- Identificaron las diferencias sobre la obtención de esfuerzos en estructuras isostáticas (acero laminado) e hiperestáticas (hormigón armado). 
- Identificaron la necesidad del uso de un programa informático para la obtención de esfuerzos en una estructura hiperestática.

- Identificaron la simplificación que supone el uso de un programa informático para la obtención de esfuerzos en una estructura isostática.

Una vez obtenidas estas conclusiones, se facilitó a los alumnos un programa informático con el que se obtuvieron esfuerzos y deformaciones en la estructura diseñada. Asimismo, se dieron nociones básicas sobre el funcionamiento de dicho programa para los fines comentados.

Es importante recalcar, que dada la dificultad presentada por los alumnos en la obtención de esfuerzos (competencia adquirida en la asignatura Estructuras I) y dado el resultado obtenido del cuestionario de evaluación inicial del alumnado, los profesores de la asignatura decidieron organizar un seminario de refuerzo sobre esta actividad fuera del horario de clases y de asistencia voluntaria. Dicho seminario presentó una asistencia masiva en las dos ediciones (mañana y tarde) que se realizaron.

\section{Taller 3: Interpretación de la normativa}

Uno de los problemas principales con los que se encuentra el estudiante es la dificultad que supone la comprensión e interpretación de la normativa vigente. Es por este motivo, que uno de los talleres planteados tenía como objetivo forzar a los alumnos a leer, entender e interpretar la normativa vigente. Concretamente, se escogió el artículo de la Instrucción de Hormigón Estructural EHE 08 (Fomento, 2008) correspondiente al cumplimiento de las limitaciones de deformaciones dentro de los Estados Límite de Servicio. El desarrollo de la clase fue tal como sigue:

- Se insta a los alumnos a que lean de una manera comprensiva el capítulo indicado de la normativa. 
- Se inicia un debate en el que se tratan:

- Relación entre el canto / deformaciones

- Relación entre el canto de forjado / canto de las vigas planas / deformaciones en ambos

- Limitaciones de la normativa

- Comprobaciones a realizar según el tipo de flecha

- Comprobación de flechas utilizando programas informáticos

- Finalmente, se aplican, con la orientación del profesor, las comprobaciones normativas a las barras del llamado proyecto de curso.

Los resultados en términos de aprendizaje de los alumnos obtenidos en esta actividad se valoraron mediante un cuestionario que se pasó antes y después de la clase presencial. Este cuestionario incluía una serie de ítems que el alumno debía marcar en función de la premisa: "Marcar los factores que influyen en la deformación de una viga". Entre la lista de factores se incluian factores de tres tipos: factores cuya influencia en la deformación es obvia (carga que afecta a la viga y longitud de la misma); factores que influyen en la viga tal como han visto en Estructuras I (inercia de la sección transversal y propiedades mecánicas de los materiales); y por último, determinados factores cuya influencia en las deformaciones no se puede detectar si no es porque se ha comprendido perfectamente el tema (fisuración y edad del hormigón). Los resultados se encuentran en la figura 6.

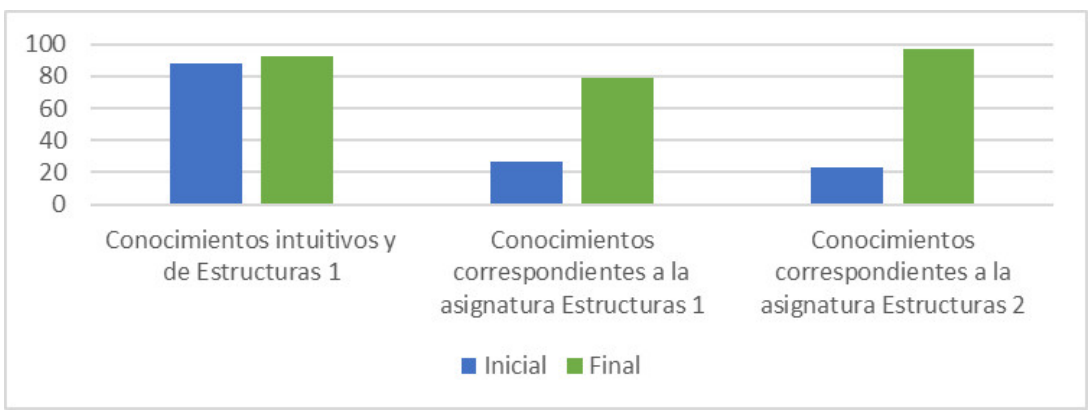

Figura 6. Porcentaje de alumnos con determinados conocimientos antes y después de la realización del taller 3.

Jornadas de Formación e Innovación Docente del Profesorado | № 2 (2019) Esta obra se distribuye con la licencia Creative Commons 
Los resultados obtenidos de la comparación de ambos cuestionarios reflejaron un importante nivel de asimilación de contenidos. Nuevamente, los alumnos presentan dificultad a la hora de relacionar conceptos abstractos de la asignatura Estructuras I con la solución a determinados problemas prácticos de la asignatura Estructuras II. Es sorprendente como, el hecho de leer e interpretar un texto denso y dificultoso como es la normativa de hormigón estructural, les ha resultado facilitador en ese aspecto. Obviamente, los resultados obtenidos en este taller han sido totalmente satisfactorios.

\section{Taller 4}

El taller 4 transcurrió en la séptima semana de clase. Ocupó los dos días lectivos de esa semana. Realmente fueron dos talleres, que transcurrieron de manera paralela y análoga, uno sobre hormigón armado y otro sobre acero laminado, es decir, uno por cada bloque temático en que la asignatura está dividida. Consistió en una sesión de trabajo autónomo de los alumnos con la orientación del profesor. En el taller 4 se testó la eficacia del nuevo enfoque del curso planteado en este ciclo de mejora de asignatura completa. Durante esta actividad se puso en común el estado de los distintos proyectos de grupo. Para ello, a los alumnos se les facilitó una lista de comprobación de los elementos que el proyecto debía incorporar. Los alumnos, en grupo, primero leyeron comprensivamente la lista de requerimientos e identificaron los que habían ya abordado, los que estaban pendientes, y los que se les habían quedado totalmente atrás. Durante las horas presenciales que duraron estos talleres, los alumnos y grupos de alumnos pusieron en común su trabajo con la orientación de la profesora.

Dado que los contenidos abarcados en el taller 4 se correspondían con todo lo trabajado hasta el momento, la evaluación del taller realmente es una evaluación del 
curso hasta el momento de su celebración. Al finalizar cada una de las dos partes del taller (hormigón armado y acero laminado) los alumnos fueron invitados a completar un test que incluía diversas cuestiones de respuesta muy sencilla, pero que englobaba todos los aspectos que habían sido tratados hasta el momento. Por tanto, también sirvió a los estudiantes para que tuvieran información sobre el estado de su aprendizaje. Los resultados de estas encuestas están en la figura 7.

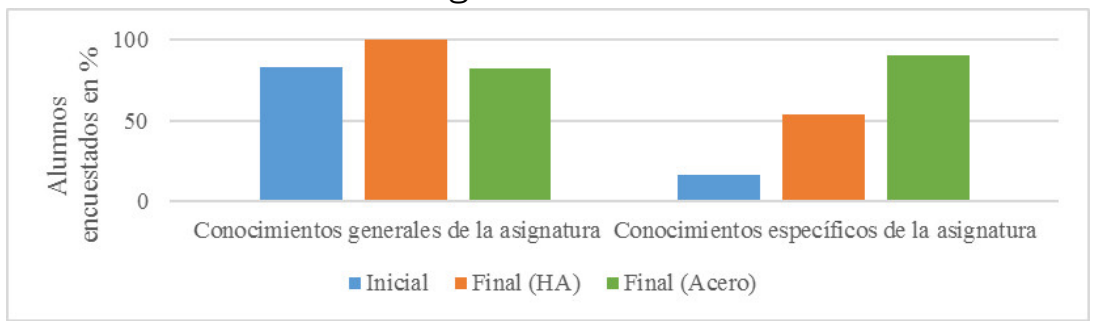

Figura 7. Porcentaje de alumnos que muestra asimilación de contenidos generales o intuitivos y otros propios de la asignatura al principio del curso y después del taller 4.

En general, los alumnos muestran buena intuición y lógica en lo que a la asignatura se refiere. Esto viene explicado por el hecho de que ya han trabajado los materiales hormigón y acero en otras asignaturas desde un punto de vista diferente al del cálculo estructural. Paralelamente, los pocos conocimientos específicos de partida (solamente aquellos alumnos que están repitiendo) con que los alumnos cuentan han sido incrementados notablemente en el momento de la realización del test. Este incremento, sensiblemente superior en el bloque temático II (Acero) que en el I (Hormigón armado), deja patente la mayor dificultad que presenta esta parte del temario para los alumnos.

\section{Evaluación del Ciclo de Mejora}

Se han utilizado varias fuentes para la evaluación del ciclo de mejora llevado a cabo. La primera de ellas, fundamental, aunque poco reglada y científica, es el feedback continuo entre los alumnos y la profesora. Desde el 
principio de curso se estableció un clima de confianza mutuo en el que los alumnos han ido dando sus impresiones sobre los temas tratados, necesidad de profundización, etc. Gracias a este feed-back, las clases se han ido reorientando en función de las necesidades planteadas.

El ciclo de mejora en si se evaluó aproximadamente transcurrida la mitad del mismo (séptima semana) mediante un formulario que incluía la valoración del profesor y la de la organización de la asignatura (Figura 8), y a su vez, estos datos comparados con los obtenidos para los dos cursos académicos inmediatamente anteriores. Pocas diferencias pueden observarse de esta comparación en lo que refiere a estas dos cuestiones

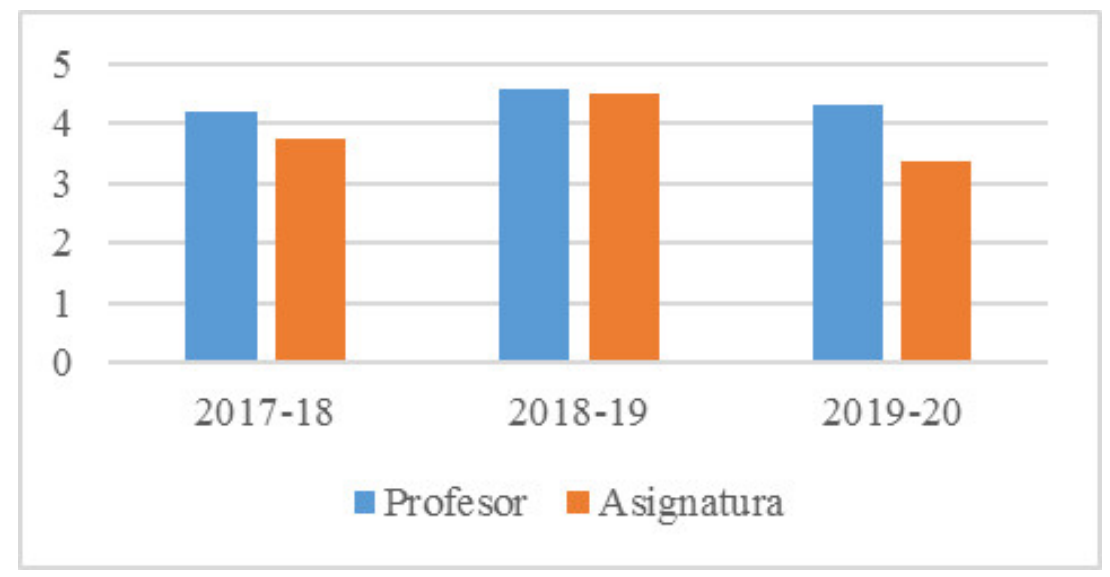

Figura 8. Evaluación del profesor y de la asignatura durante los tres últimos cursos académicos.

Adicionalmente, de estas encuestas se desprendió que el $70 \%$ de los alumnos encuestados considera el nivel de dificultad de la asignatura medio-alto y el $25 \%$ el temario demasiado extenso. Paradójicamente, casi el 50\% de los alumnos confiesa dedicar a la asignatura menos de las 6 horas de trabajo no presencial contempladas en el programa. 


\section{Conclusiones}

En este documento se ha recogido el resultado de una experiencia llevada a cabo sobre la totalidad de una asignatura de las que los alumnos califican como "dificiles". Esta asignatura, dividida en "teoría" y "práctica", presentaba la dificultad por parte de los alumnos de vincular ambas, relacionando la una con la otra. El rediseño total de la asignatura ha tratado de solventar ese salto, organizando ambas en torno al mismo eje vertebrador, es decir, un proyecto de arquitectura. En general, los resultados obtenidos comparando las evaluaciones previas y final del alumnado han sido bastante satisfactorios, pues el nivel de asimilación de conceptos se ha facilitado en gran medida. Paradójicamente, las evaluaciones por parte de los alumnos a la asignatura han sido similares a las realizadas en cursos anteriores. El aparente motivo del mismo podría ser la concepción del alumno de la asignatura como extensa y dificil, evaluando exclusivamente lo que se hace durante las clases presenciales. Prueba de esto es el elevado porcentaje de alumnos encuestados que ha dedicado menos horas no presenciales a la asignatura de las establecidas en el programa de la misma.

Jornadas de Formación e Innovación Docente del Profesorado | № 2 (2019) Esta obra se distribuye con la licencia Creative Commons 


\section{Referencias bibliográficas}

Alison Burke. (2011). Group work: How to use groups effectively. The Journal of Effective Teaching, 11(2), 87-95.

AtelierRISTING. (2018). Glass Cabin. Retrieved November 5, 2019, from http://www.atelierristing.com/glass-cabin.

Bain, K., \& fesores de universidad. Universitat de València.

España. Ministerio de la Vivienda. (2011). Código Técnico de la Edificación. Retrieved from codigotecnico.org

Finkel, D. L. (2000). Teaching with your mouth shut. Boynton/Cook Publishers.

Fomento, M. de. (2008). Instruccion de Hormigón Estructural EHE.

Kozlowski, S. W. J., \& Ilgen, D. R. (2006). Enhancing the effectiveness of work groups and teams. Psysicological Science in the Public Interest, 7(3), 77-124.

Porlan, R. (2017). Enseñanza Universitaria. Cómo mejorarla. (Noviembre). Sevilla: Morata.

Rodríguez-Mayorga, E., Jiménez-Alonso, J. F., Pachón-García, P., Cámara, M., \& Compán, V. (2018). Conceptual workshops for the learning of architectural structures. In L. Gómez-Chova, A. López-Martínez, \& I. Candel-Torres (Eds.), ICERI2018 Proceedings. IATED Academy. https:// doi.org/978-84-09-05948-5

Rodriguez Mayorga, M. E. (2018). ¿Cómo calcular una estructura? Nuevos planteamientos docentes. Jornadas De Formación E Innovación Docente Del Profesorado, (1), 637-654. https://doi.org/10.12795/jdu.2018.i01.36

S-AR. (2015). Casa Caja. Retrieved from https://arqa.com/ arquitectura/casa-caja-en-mexico.html

Jornadas de Formación e Innovación Docente del Profesorado | № 2 (2019) Esta obra se distribuye con la licencia Creative Commons Reconocimiento-NoComercial-SinObraDerivada Internacional (CC BY-NC-ND 4.0.) 